\title{
Punctate sensitivity of normal and frostbitten skin
}

\author{
F. B. COLAVITA, S. BINGAMAN, D. DEVOS AND R. TKAK \\ UNIVERSITY OF PITTSBURGH
}

Two equal areas of normal and frostbitten human skin were compared for the ir punctate sensitivity to warmth, cold, touch, and pressure. The frostbitten tissue was characterized by a decreased sensitivity to the cold stimulus and by a tendency for warm and touch stimuli to elicit reports of pain from the $S$. There appeared to be no differences in pressure sensitivity between the normal and damaged tissue. Some implications of the data for somesthetic sensitivity in general are mentioned.

Considerable controversy exists regarding the nature of the cutaneous sense modality. The extreme positions in this controversy are the "specific receptor" view emanating from the work of von Frey (1895), and the "communality" view as expressed by Weddell and his co-workers (1955). In brief, the specific receptor position proposes that there are four distinct skin senses, warmth, cold, pressure, and pain, each subserved by separate receptors and neural pathways. The communality view maintains that there are no specific receptors or pathways, but rather a unitary system that allows an organism to discriminate between different qualities of cutaneous inputs on the basis of complex temporal and spatial patterns of neural impulses.

An approach has recently been proposed by Melzack \& Wall (1962) that represents a synthests of the best features of both specificity theory and pattern theory. Data obtained in the present experiment, comparing the punctate sensitivity of normal and frostbitten skin in a human $S$, are in accord with the position of Melzack and Wall.

Method

The $S$ was a 24 yearold male who had been frostbitten on the left cheek at the age of 20. The injury had been diagnosed as frostbite by a physician and fulfilled the criteria for bona fide frostbite mentioned by Washburn (1963).

The apparatus used consisted of a wire von Frey hair for touch, warm and cold temperature probes, and a pressure stick. The von Frey hair was .005 in. in diameter and exerted a force of $.5 \mathrm{~g}$. The cold stimuli were four brass cylinders, 3 in. long and $1 / 2$ in. wide. The cylinders were conical at one end with a tip diameter of $1 \mathrm{~mm}$. The brass cylinders were maintained at a temperature of $24^{\circ} \mathrm{C}$ in an ice and water bath. The four cold stimuli were alternated in use so that the effective stimulus temperature was always at $24^{\circ} \mathrm{C}$. The warm stimulus was an Ungar No. 1237 heating unit with a tip diameter of $1 \mathrm{~mm}$. The heating unit was warmed to $44.6^{\circ} \mathrm{C}$ by delivering an appropriate voltage to it from a Simpson variac. Temperature was monitored continuously with a Digitec model 4134 digital readout thermom- eter. The wooden stick used for pressure stimulation had a tip diameter the same size as that of the warm and cold stimuli.

A $2 \mathrm{sq} \mathrm{cm}$ section of frostbitten tissue located $2 \mathrm{~cm}$ below S's left eye was divided into $1002 \mathrm{sq} \mathrm{mm}$ spots with a rubber grid stamp. An equal area of normal tissue was marked off on the right cheek for comparison purposes. The areas to be tested were closely shaved prior to the start of the experiment.

Each grid square was then tested by being lightly touched in the center with one of the stimulus objects. The $S$, without visual cues and lacking information as to which stimulus had been presented, was asked to report on the quality of the sensation he experienced. Every square on both cheeks was tested twice with each type of stimulus.

\section{Results and Discussion}

The verbal reports of $S$ are shown in Table 1. No instances of paradoxical cold or heat were encountered. Points touched with the temperature stimuli where there was no report of warmth or cold were usually experienced by $S$ as pressure.

An obvious feature of the present data is the relative insensitivity of the frostbitten tissue to the cold stimulus. Only two frostbitten spots yielded sensations of cold on both mappings. In contrast to this, approximately $60 \%$ of the spots on the normal skin produced cold sensations on both mappings.

The number of spots responsive to the warm stimulus was about the same for the normal and damaged tissue.

\section{TABLE 1}

First and Second Mappings of Punctate Sensitivity for Normal and Frostbitten Skin

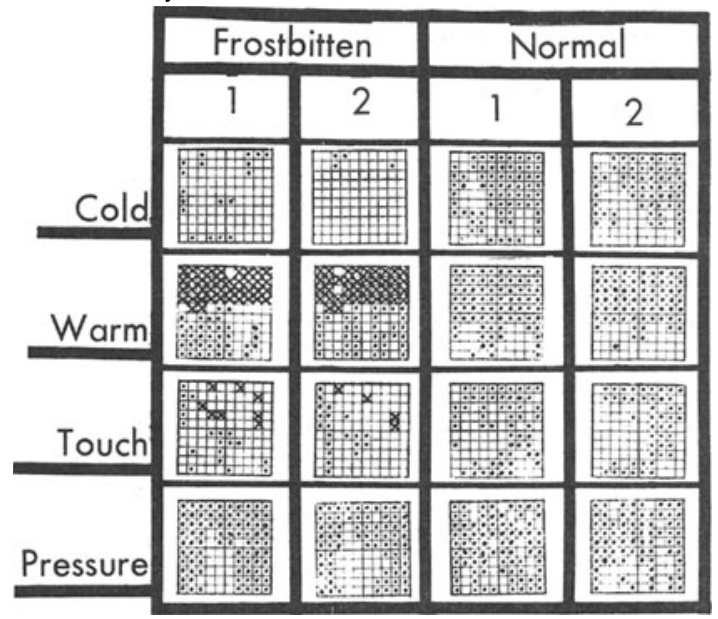


The difference was in the quality of the resulting sensation. Those grid squares indicated by an $\mathrm{X}$ in Table 1 elicited reports of "painful" from the S. Table 1 shows that over half of the warm spots on the frostbitten tissue are associated with such a report while no such spots are to be found on the normal tissue.

Touch sensitivity showed a double aspect alteration in the frostbitten tissue. Not only were there fewer touch spots on the damaged skin relative to the normal tissue, but approximately $1 / 3$ of the touch-sensitive frostbitten spots were associated with reports of pain (indicated by $X$ in Table 1). Such an effect was not observed with the normal skin. Finally, Table 1 shows that there was no apparent change in pressure sensitivity as a result of frostbite.

The differential changes in cutaneous sensitivity seen in the present experiment suggest the involvement of peripheral nerve damage in the process of frostbite; however, existing data do not support this notion. It is apparently quite difficult to produce irreversible changes in nerve tissue through cold exposure. Miller (1965) has found that excised peripheral nerves in five varieties of mammals continued to exhibit neural activity when subjected to temperatures as low as $-6^{\circ} \mathrm{C}$. In some cases spontaneous freezing of the tissue began before the neural activity stopped. If rewarming of frozen or partially frozen nerves was begun shortly after complete disappearance of the action potential, full recovery of function was seen to occur.

Generalizing from animal nervous tissue to human nervous tissue (as is typically done), it appears that the sensitivity differences between the normal and frostbitten tissue observed in the present study are not attributable to permanent changes in the conduction properties of peripheral nerves. This supposition is reasonable in that the conditions leading to frostbite in $S$ were not as severe as those reported to be necessary for permanent peripheral nerve changes (see Ariev, 1940).

If there were no permanent changes in the firing characteristics of peripheral nerves, classical pattern theory, which contains no provisions for different kinds of receptors, cannot account for the presentdata. Similarly, classical specificity theory would have difficulty in explaining the above data in terms of modality-specific receptors and pathways, each carrying information about one and only one kind of stimulus. Why should cold receptors be affected and not pressure receptors, when both are supposedly located in the more superficial skin layers? And how is it that warmth and touch stimulation sometimes produce sensations of pain? Questions of this type cannot be answered by specificity theory.

As mentioned earlier, the present data seem to be in accord with the approach of Melzack \& Wall (1962). Melzack and Wall propose that there are specialized receptors, but not in the classical sense. Rather, there are receptors specialized physiologically for the transduction of particular kinds and ranges of stimuli into patterns of nerve impulses. These investigators further propose that every discriminably different cutaneous perception is produced by a unique pattern of nerve impulses that can represent the combined outputs of different receptors with some overlap in their specificity ranges.

The significance of this line of reasoning for the above data is clear. If frostbite were to alter the characteristics of certain receptors made especially vulnerable by their particular physiological specificity, the neural output characteristic of these particular receptors in response to a given stimulus would be changed. This, in turn, could alter the subjective experience of the frostbite victim for all qualities of cutaneous stimulation that formerly fell within the range of physiological specificity of the affected receptors. Since the unique pattern of nerve impulses associated with a given cutaneous stimulus may be derived from various combinations of the outputs of receptors affected by frostbite and receptors unaffected by frostbite, some subjective experiences might be diminished, some might be changed qualitatively, and some might be unaffected.

Remaining to be discovered are the mechanisms by which frostbite can effect alterations in cutaneous receptors, and the characteristics of certain receptors that make them susceptible to frostbite damage. The present data suggest that a better understanding of the receptor changes accompanying frostbite will increase our understanding of normal somatosensory sensitivity.

\section{References}

Ariev, T. J. Monograph on frostbite. (Translated by 1. Steiman). Narkomzdrav, U.S.S.R., State Health Committee, 1940.

Melzack, R., \& Wall, P. On the nature of cutaneous sensory mechanisms. Brain, 1962, 85, 331-356.

Miller, L. K. Activity in mammalian peripheral nerves during supercooling. Science, 1965, 149, 74-75.

von Frey, M. Beiträge sur Sinnesphysiologie des Haut. Ber Sächs Ges. Wiss. Math-Phys. Cl., 1895, 47, 166-184.

Washbum. B. Frostbite-What it is-How to prevent it-Emergency treatment. Bosto., Museum of Science, 1963.

Weddell, G. Somesthesis and the chemical senses. Annu. Rev. Psychol., 1955, 6, 119-136. 